\title{
The invasion history of Microsphaera palczewskii (Erysiphales) in Finland
}

\author{
SEPPO HUHTINEN, PENTTI ALANKO and YRJÖ MÄKINEN
}

\begin{abstract}
HUHTINEN, S., ALANKO, P. \& MÄKINEN, Y. 2001: The invasion history of Microsphaera palczewskii (Erysiphales) in Finland. - Karstenia 41: 31-36. ISSN 0453-3402.
\end{abstract}

The invasion of Microsphaera palczewskii Jacz. - an Asian-origin parasitic fungus of Caragana Fabr. - in Finland is reported. The first specimen was collected in South Finland in 1981 and now the species is distributed throughout the country to all areas where Caragana arborescens Lam. is cultivated. The epidemic spread caused a total decline of the occurrence of $M$. trifolii (Grev.) U. Braun on Caragana. The last record of $M$. trifolii dates back to year 1987. Mature cleistothecia of $M$. palczewskii can be found from mid-July onwards, conidial stage from the beginning of June. Specimens showing conidial stage only can be observed even in September and October, which indicates a continuous infection activity. Recent field observations do not support the reported overwintering in wooden parts. The source of infection is most probably fallen leaves with plenty of cleistothecia.

Keywords: Microsphaera, Caragana, Finland, invasion history

Seppo Huhtinen and Yrjö Mäkinen, Herbarium, University of Turku, FIN-20014 Turku, Finland

Pentti Alanko, Korsutie 34, FIN-00370 Helsinki, Finland

\section{Introduction}

The characteristics, occurrence and epidemic spread of Microsphaera palczewskii Jacz. (Erysiphales) have recently been quite well documented. The species originates in Asia (Russian Far East and China to Kazakhstan) and has since 1975 also been known in Europe. Much has been written on its invasion history and to the list provided by Braun (1995) and Scholler (1994a, 1994b) the following new treatises can be added: Eriksson (1992), Gjærum (1994), Kreisel \& Scholler (1994), Karis (1995), Gelyuta (1997), Tikhomirova \& Tobias (1999). As summarized by Scholler (1994a), the species was first discovered in Europe in 1975. The first collection originates from Belarus. The next finds were made in all directions from this area. The distribution map pub- lished by Scholler (1994a, 1994b) contained many records from Finland provided by Prof. Teuvo Ahti. According to the map M. palczewskii appeared to be most abundant in Finland. The expansion pattern led Scholler (1994b) to the conclusion that $M$. palczewskii did not gradually widen its area westward from its original area, but was sporadically first introduced to Europe in some places in Belarus or in the Baltic Republics. Since the mid-seventies, the species gradually moved to all areas where its hosts - species of the genus Caragana Fabr. (Fabaceae) - are cultivated as ornamental plants. Its northern limit has now reached $68^{\text {th }}$ parallel in Finland. The spread appears irreversable and the species is a typical neomycete (Kreisel \& Scholler 1994) in Europe. 


\section{Materials and methods}

All the Finnish specimens of powdery mildews on Caragana in the major Finnish herbaria (H, OULU, TUR) were checked. Also the sheets of Caragana in the phanerogamic collections of $\mathrm{H}$ and TUR were checked. Determinations are almost exclusively based on the presence of mature or nearly mature cleistothecia, bearing in mind also Microsphaera coluteae Komarov (Braun 1987). Due to the reported difficulty of separating the different species by their anamorph (Scholler 1994a), collections showing only conidial formation are cited with an asterisk in the list of studied material. Due to the overall efficiency of the invasion of $M$. palczewskii, they most probably also represent this species, being mostly collected well after the last definite records of $M$. trifolii.

\section{Phenology}

The first specimens of $M$. palczewskii that show only surface mycelium and conidial formation have been collected during the first week of June in South Finland. Immature specimens dominate to the middle of July. Cleistothecia begin to mature towards the end of July in South and Central Finland. In August and September the species is easily visible; hence the peak of collections occurs during these two months (Fig. 1). There seems to be no greater difference in phenology between South and North Finland, except that there are no collections after mid-September from the North. Specimens with only the conidial stage have occasionally been collected also in September and October. This parallels the observations by Scholler (1994a), who reported juvenile asci also in material collected at the end of October (in N Germany).

\section{The spread}

Before the arrival of M. palczewskii in Finland, Caragana arborescens was practically free of heavy infections by powdery mildews. However, the polyphagous M. trifolii (Grev.) U. Braun (syn. Erysiphe trifolii Grev.) was occasionally found on this host. The arrival of another species, specialized on this host, changed this situation drastically. The first specimen of M. palczewskii was collected in Helsinki in 1981. The species was first reported in Finland by Hämet-Ahti et. al. (1992). By the end of year 1981 M. trifolii had been observed eleven times, at seven localities in South Finland (Fig. 2). By 1983 two new localities for M. palczewskii were observed, both still in South Finland. M. trifolii was still collect-

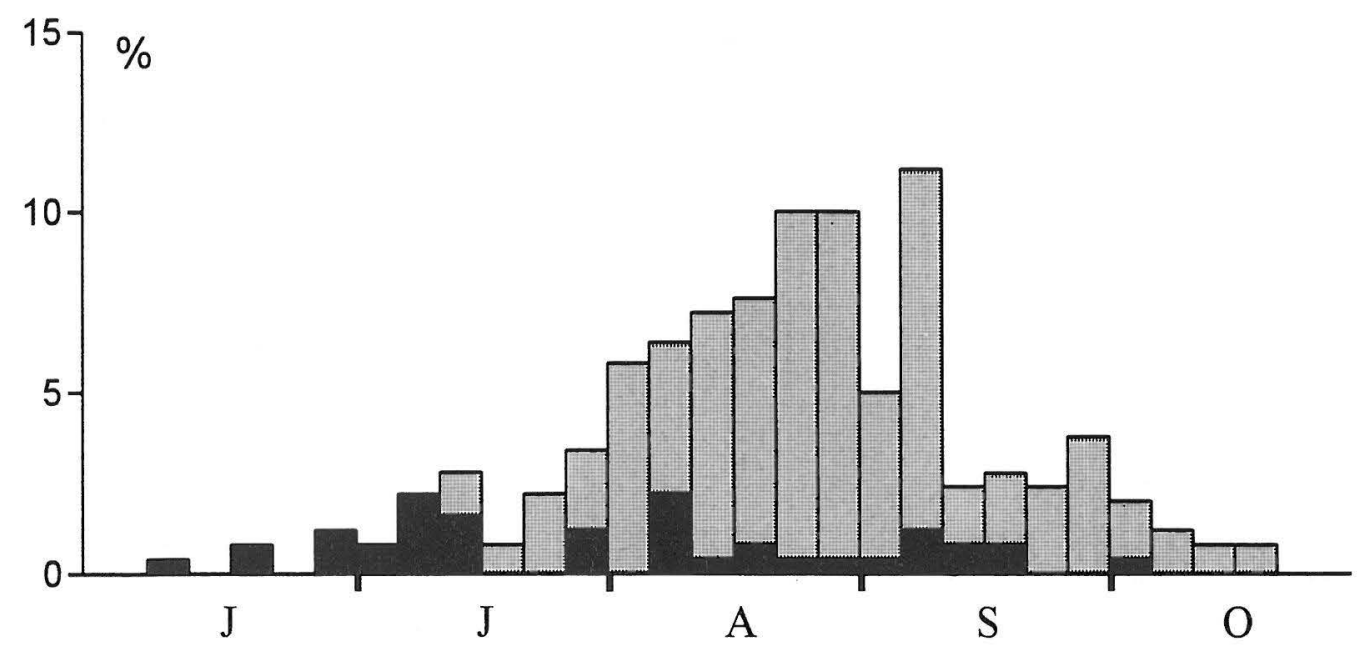

Fig. 1. The percentual distribution of 240 Finnish collections of Microsphaera palczewskii according to collection dates from June to October, divided in pentads. Blackened part indicates juvenile specimens (with conidia). 

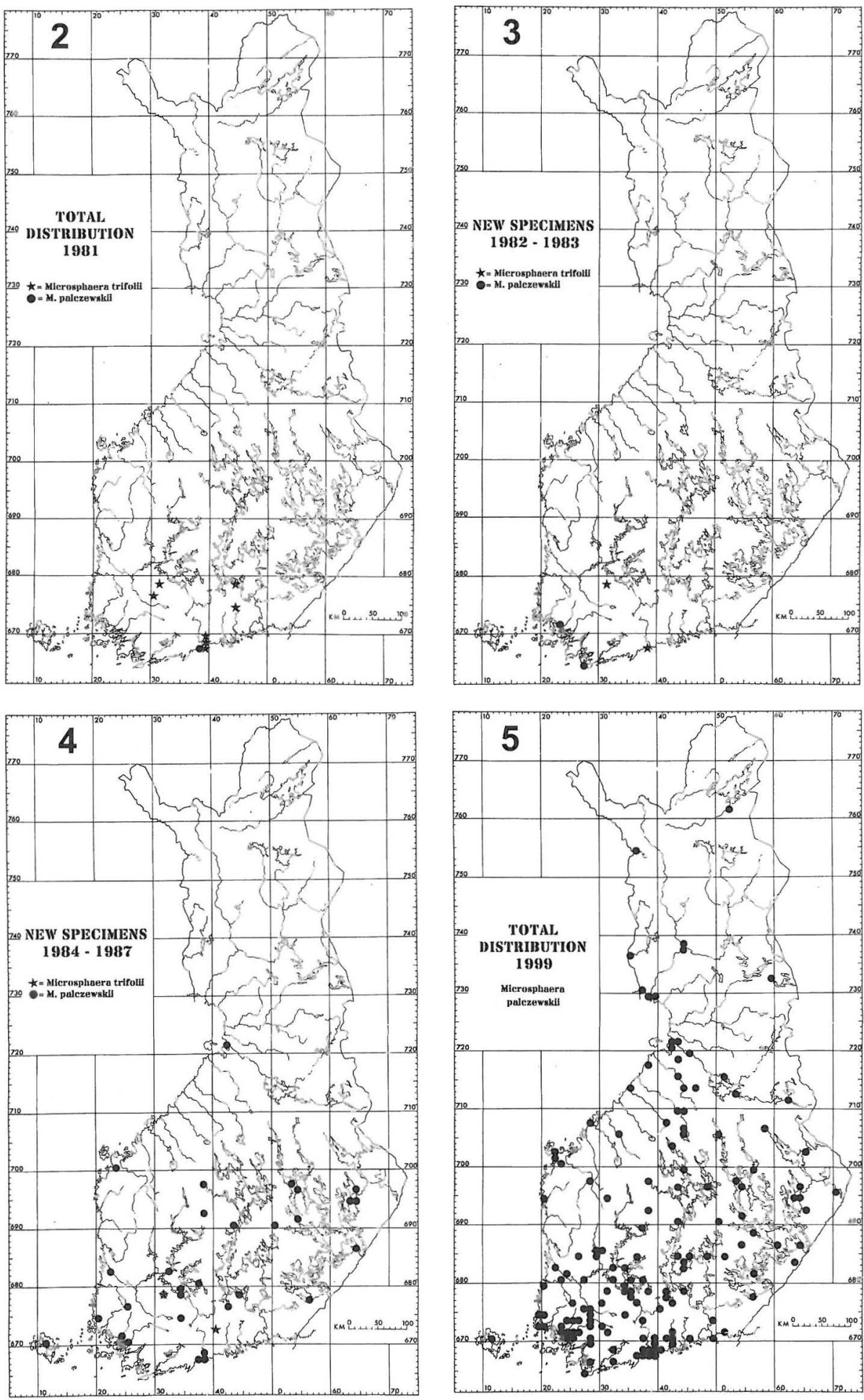

Figs. 2-5. The invasion of Microsphaera palczewskii into Finland and the simultaneous decline of M. trifolii from the same host. 
ed twice on Caragana; one locality being a new find (Fig. 3). By 198514 new collections of $M$. palczewskii were made and the species had now spread to Central Finland. At the same time only one specimen of $M$. trifolii was found in Hattula; a site where the species had persisted on Caragana from the year 1965. By 198717 new specimens of M. palczewskii had been collected and the species had now reached Oulu in northern Finland (Fig. 4). The year 1987 marked also the last collection of $M$. trifolii on Caragana. By the end of 1998 M. palczewskii had reached Ivalo and Muonio in Lapland.The present distribution is shown in Fig. 5. The fast invasion is explained by the unusual abundance of $C$. arborescens as an ornamental plant in Finland (Hämet-Ahti et. al. 1992). Caragana arborescens was apparently first introduced to Europe in 1727, when D. Messerschmidt brought seeds to St. Petersburg from his expedition to Yenisey River, Siberia (Buligin \& Firsov 1998). According to Parvela (1930) seeds of Caragana were brought from St. Petersburg to Turku, Finland, in 1744, where the plants produced their first flowers in 1748 (Bjelke 1750). C. arborescens was similarly introduced to many other countries in Europe from Russia. C. frutex was brought into cultivation in St. Petersburg in 1736 through seeds collected by I. Heintzelmann (Buligin \& Firsov 1998), probably along the Volga River in SE Russia. It never became as widespread in Finland and elsewhere in Europe as $C$. arborescens.

A search for M. trifolii in 1998 from the same shrubs on which it had occurred for 17 (Helsinki, Malmi) and 20 (Hattula, Lepaa) years showed that the species could not be found any more (observations by P.A.). Leaves from basal shoots were especially observed, because on these the more massive infection of M. palczewskii was not as likely to mask the occurrence of $M$. trifolii. It is likely that $M$. palczewskii, being a newly arrived specialist on this genus, has actually ousted the other species with weaker and more occasional infection on Caragana.

M. palczewskii often causes so heavy infections that even free-growing shrubs of Caragana become conspicuously white. Especially severe infections can be observed in hosts grown in hedges, which are regularly cut and thus develop a continuous dense "monoculture" of even-aged shoots. Due to this fact, some gardeners have replaced hedges of $C$. arborescens with other species. As to be expected, the fungus seems to make no difference between different cultivars of Caragana. Twenty specimens of $C$. arborescens 'Lorbergii' and 'Pendula' have been collected from mid-August to the end of September, their phenology apparently reflecting overall collection activity. Only one specimen originates from $C$. frutex, on which species $M$. palczewskii has not been previously reported (cf. Karis 1995).

Scholler (1994a) reported that M. palczewskii most likely overwinters as mycelium inside wooden parts of the host. To support this he had a series of in situ observations where the first signs of surface mycelium developed at the basal parts of the leaflets. A study of 30 juvenile specimens from Finnish herbaria showed no support for this idea. Also field observations verified that the first mycelial patches may develop to any part of the leaf and leaflet. In one collection mycelium and cleistothecia developed on empty pods of $C$. arborescens.

\section{Material studied}

\section{Microsphaera trifolii}

Uusimaa: Artjärvi, 1981 Haikonen $1674(\mathrm{H})$, Helsinki, 1966, 1971 Savas 4, 879 (H), 1972, 1983 Alanko 23144, 23308, 23326, $44996(\mathrm{H}, 23144$ also in OULU, TUR). Mäntsälä, 1987 Haikonen 8864 (H); Vantaa, 1966, 1971 Savas, 101, 963 (H). Etelä-Häme: Hattula, 1965, 1976, 1982, 1985 Alanko 32792, 43774, $53602(\mathrm{H}), 67835,410-65$ (OULU). Heinola, 1954 Rauhala (H). Humppila, 1972 Alanko $21929(\mathrm{H})$.

M. palczewskii (those showing only conidia marked with *) Ahvenanmaa: Sund, 1984 Mäkinen 84-874 (TUR). Varsinais-Suomi: Aura, 1989 Mäkinen 89-1212 (TUR). Halikko, 1998 Kapanen \& Kosonen* (TUR). Kaarina, 1989, 1993 Mäkinen 89-863*, 93-1527 (TUR). Karinainen, 1997 Huhtinen (TUR). Karjalohja, 1999 Alanko 104045 (H). Karkkila, 1997 Alanko 94089 (H). Kiikala, 1995 Kapanen \& Kosonen (TUR). Koski, 1991 Kapanen; 1996, Mäkinen \& Heinonen \& Heinonen 44-96*; 1998 Kapanen \& Kosonen* (TUR). Kustavi, 1999 Kallio (TUR). Marttila, 1998 Kapanen \& Kosonen * (TUR). Paimio, 1991 Kapanen (TUR); 1998 Kapanen \& Kosonen* (TUR). Perniö, 1994 Kapanen \& Kosonen (TUR). Piikkiö, 1986 Alanko 57110 (H); 1998 Kapanen \& Kosonen (TUR). Raisio, 1998 Kapanen \& Kosonen (TUR); 1999 Kallio (TUR). Rusko, 1998 Kapanen \& Kosonen (TUR). Salo, 1994, 1997 Kapanen \& Kosonen (TUR). Taivassalo, 1989 Laine (TUR). Tammisaari, 1998 Alanko 99212 (H). Turku, Kallio 1999 (TUR); 1983, 1986, 1989, 1993, 1996 Alanko 46783, 57243, 66437, 76389, 90061, $90079(\mathrm{H})$; 1983*; 1984, 1994 Mäkinen 83-1475*, 841425, 88-754*, 94-503, 94-532, 94-1010 (TUR); 1993, 1994 Kapanen, 1996*, 1998* Kapanen \& Koso- 
nen (TUR). Vahto, 1998 Kapanen \& Kosonen (TUR). Vehmaa, 1984 Mäkinen 84-1222 (TUR). Uusikaupunki, 1993 Mäkinen 93-1161 (TUR). Västanfjärd, 1998 Alanko 97857 (H). Uusimaa: Askola, 1996 Höijer 2021 (TUR). Espoo, 1984, Ahti 41887 (H); 1990 , 1994, 1996, 1998 Alanko 68349, 80247, 89930, 98858 (H). Hanko, 1983 Alanko 46965 (H); 1991 Kapanen \& Kosonen (TUR). Helsinki, 1981, 1984, 1989, 1993, 1994, 1995, 1996, 1997, 1999 Alanko 41916, 49549, 49590, 49593, 50378, 65826, 76772, 79421, 79649, 83493, 89192, 90965, 93874, 94441-94444, $100720(\mathrm{H}) ; 1991$ Ahti $50940(\mathrm{H}) ; 1993 \operatorname{Harju}(\mathrm{H})$; 1995 Mäkinen 95-389 (TUR); 1995 Gustafsson (H); 1996 Höijer 2020 (TUR); 1997 Saari (TUR). Järvenpää, 1992 Höijer 882 (TUR). Kerava, 1996 Höijer 1952 (TUR). Kärkölä, 1997 Alanko 94629 (H). Loviisa, 1996 Höijer 2014 (TUR). Nurmijärvi, 1989 Askola 2654 (H). Pornainen, 1996 Kapanen \& Kosonen (TUR). Porvoo, 1996 Höijer 1935 (TUR); 1997 Alanko 93930 (H). Sipoo, 1996 Höijer 2019 (TUR). Vantaa, 1991 Joutsenlahti 189* (TUR). Etelä-Karjala: Anjalankoski, 1994 Kapanen \& Kosonen (TUR). Hamina, 1997 Alanko 94181 (H). Kotka, 1998 Höijer 2628 (TUR). Satakunta: Harjavalta, 1984 Mäkinen 841138* (TUR). Hämeenkyrö, 1992 Alanko 73130 (H). Ikaalinen, 1993*, 1996 Kapanen \& Kosonen (TUR). Kiukainen, 1996 Kapanen \& Kosonen (TUR). Lavia, 1998 Kapanen \& Kosonen (TUR). Loimaa, 1991 Kapanen (TUR). Loimaan mlk, 1993 Kapanen (TUR). Mellilä, 1998 Kapanen \& Kosonen* (TUR). Pori, 1985 Alanko 53849, 53870 (H). Rauma, 1992 Ahti 51338 (H). Yläne, 1984 Mäkinen 84-1105 (TUR). Äetsä, 1996 Kapanen \& Kosonen (TUR). Etelä-Häme: Asikkala, 1984, 1989 Haikonen 4789, 10985 (H). Hartola, 1993 Alanko $75529(\mathrm{H}) ; 1994$ Haikonen $16588(\mathrm{H}) ; 1997$ Höijer 2265 (TUR). Hattula, 1984, 1996, 1997 Alanko 49047, 90880, 94047 - 94051 (H); 1997 Höijer 2262 (TUR). Heinola, 1986 Alanko 57330 (H). Hollola, 1989 Alanko $66482(\mathrm{H}) ; 1991$ Haikonen $13303(\mathrm{H})$; 1995 Kapanen \& Kosonen* (TUR). Hämeenlinna, 1990 Ahti 49083 (H). Iitti, 1984 Haikonen 5067 (H). Kangasala, 1995 Mäkinen 95-347 (TUR). Lahti, 1986 Alanko 57316 (H); 1995, Kapanen \& Kosonen* (TUR). Lempäälä, 1993, Alanko 76108 (H); 1996 Söderholm 2544 (TUR); 1998 Nummela-Salo \& Salo 6543 (H, TUR). Luopioinen, 1984 Alanko 49035 (H). Orivesi, 1995 Kosonen (TUR). Pälkäne, 1988 Haikonen 9815 (H). Riihimäki,1995 Ahti 52634 (H). Somero, 1993 Kapanen (TUR), 1997 Alanko 94654 (H). Tampere, 1986, 1996 Alanko 57501, 90850 (H); 1994 Mäkinen 94-475 (TUR); 1998 Kapanen \& Kosonen (TUR). Tuulos, 1998 Alanko 98914 (H). Valkeakoski, 1986 Alanko 57059 (H). Vilppula, 1998 Järvinen \& Järvinen 98-566 (TUR). Etelä-Savo:Hartola, 1999 Alanko $102316(\mathrm{H})$. Hirvensalmi, 1992 Alanko 73191 (H). Joutsa, 1997 Höijer 2266 (TUR). Juva, 1988 Alanko $63506(\mathrm{H})$. Lappeenranta, 1987, 1993 Alanko 60888, 76585 (H). Mikkeli, 1988 Alanko $62558(\mathrm{H})$. Puumala, 1992 Alanko 73053 (H). Rantasalmi, 1998 Kapanen \& Kosonen (TUR). Savonlinna, 1998 Kapanen \& Kosonen (TUR). Laatokan Karjala: Parikkala, 1989 Kapanen (TUR). Etelä-Pohjanmaa: Alavus, 1996 Alanko 89435 (H). Närpiö, 1993 Kapanen (TUR). Seinäjoki,
1996*, 1998 Kapanen \& Kosonen (TUR). Vaasa, 1986 Piispala 823 (H); 1991 Kapanen (TUR); 1991 Ahti 50151 (H). Pohjois-Häme: Jyväskylä, 1986 Alanko $57596(\mathrm{H})$; 1997 Höijer 2268 (TUR). Jyväskylän mlk, 1997 Höijer 2267 (TUR). Karstula, 1986 Alanko 57669. Konginkangas, 1994 Kapanen \& Kosonen (TUR). Multia, 1985 Alanko 53834 (H). Pihtipudas, 1997 Höijer 2272 (TUR). Rautalampi, 1995 Alanko 83519 (H). Viitasaari, 1997 Höijer 2271 (TUR). Pohjois-Savo: Iisalmi, 1994 Alanko 79717 (H). Muuruvesi, 1988 Ahti 47619 (H). Kuopio, 1984 Koskela (H, OULU);1998, Alanko 99650 (H). Pieksämäki, 1986 Alanko 57361, 57394, 57395 (H). Varkaus, 1986 Alanko 57376 (H). Vehmersalmi, 1984 Alanko 48917 (H). Pohjois-Karjala: Ilomantsi, 1996 Höijer 1998. Joensuu, 1987 Alanko 60467, 60585 (H). Kesälahti, 1987 Alanko 60901 (H). Lieksa, 1994 Kapanen \& Kosonen (TUR). Pyhäselkä, 1996 Kosonen (TUR). Valtimo, 1988 Ahti 47624 (H). Keski-Pohjanmaa: Haapajärvi, 1992 Kapanen \& Kosonen (TUR). Kalajoki, 1998 Höijer 2654 (TUR). Kaustinen, 1992 Kapanen \& Kosonen (TUR). Kestilä, 1997 Höijer 2421 (TUR). Kärsämäki, 1996 Kulju 88/96 (OULU); 1997 Höijer 2274 (TUR). Pietarsaari, 1994 Alanko 80741 (H). Pulkkila, 1997 Höijer 2275 (TUR). Pyhäjärvi, 1996 Kulju 85/96, 87/96 (OULU); 1997 Höijer 2273 (TUR). Raahe, 1998 Höijer 2653 (TUR). Rantsila, 1996 Kulju 90/96 (OULU). Oulun Pohjanmaa: Kempele, 1995 Alanko 84808 (H). Muhos, 1997 Höijer 2279 (TUR). Oulu, 1989 Salo 7757 (OULU); 1986, 1989, 1991, 1992, 1995 Alanko 56427, 65893, 70596, 72944, 76389, 84735, $84737(\mathrm{H}) ; 1986,1991$ Väre (H, OULU); 1996 Kulju 72/96 - 79/96 (OULU). Tyrnävä, 1996 Kulju $86 /$ 96 (OULU); 1997 Höijer 2276 (TUR). Kainuu: Kajaani, 1997 Kosonen \& Rautiainen 97-129.(TUR). Kuhmo, 1995 Alanko $84264(\mathrm{H})$. Vaala, 1999 Alanko 101930 (H). Perä-Pohjanmaa: Kemi, 1991, anonymous (OULU); 1997 Höijer 2287 (TUR). Rovaniemi, 1995 Kapanen \& Kosonen* (TUR); 1995, 1996 Alanko 84409, 84430, 90388, 90488 (H). Tornio, 1997 Höijer 2290 (TUR). Ylitornio, 1998 Höijer 2594 (TUR). Koillismaa: Kuusamo, 1989 Ahti $48488(\mathrm{H}) ; 1996$ Alanko 90471, 90475 (H). Kittilän Lappi: Muonio, 1998 Höijer 2600 (TUR). Inarin Lappi: Ivalo, 1998 Mäkinen 98-911* (TUR).

Acknowledgements: The collectors are warmly thanked for their efforts. Prof. Teuvo Ahti has painstakingly helped us during the writing process.

\section{References}

Bjelke, S. C. 1750: Beskrifning om det Siberiska ÄrteTrädet. - K. Svenska Vet. Acad. Handl. 11: 122-127.

Braun, U. 1987: A monograph of the Erysiphales (powdery mildews).- Nova Hedwigia 89: 1-700.

Braun, U. 1995: The powdery mildews (Erysiphales) of Europe. - Gustav Fischer, Jena. 337 pp.

Buligin, N.E. \& Firsov, G.A. 1998: Few known data of I. Falck concerning introduction of some arboreal species of Russian flora into general cultivation. (In Russian). - Bot. Zhurn. 83: 85-89. 
Eriksson, O. 1992: The non-lichenized pyrenomycetes of Sweden. - SBT-förlaget. Lund. 208 pp.

Gjærum, H.B. 1994: Rødhyll- og ertebusk-mjøldogg. Blekksoppen 63: 20-22.

Gelyuta, V.P. 1997: Analysis of phylogenic relationships of species of the genus Microsphaera Lév. (Erysiphales) parasitizing legumes. - Ukr. Bot. J. 54: 512.

Gelyuta, V.P. \& Gorlenko, M.V. 1984: Microsphaera palczewskii Jacz. in the U.S.S.R. - Mikol. Fitopatol. 18: $177-182$.

Hämet-Ahti, L., Palmén, A., Alanko, P. \& Tigerstedt, P.M.A. 1992: Suomen puu- ja pensaskasvio. Woody Flora of Finland. - Publ. Finnish Dendrol. Soc. 6: 1373.

Karis, H. 1995: Erysiphaceae Lév. in Eastern Europe and North Asia. - Tallinn. 304 p.

Karis, H. \& Normet, T. 1999: Puittaimedel parasiteerivate jahulikasteliste uutest liikidest, teisenditest ja peremeestaimedest Eestis. - In: Sander, H. (ed): Den- droloogilised uurimused Eestis 1. Dendrological researches in Estonia 1: 150-154. - EAU Forest Research Institute.

Kreisel, H. \& Scholler, M. 1994: Chronology of phytoparasitic fungi introduced to Germany and adjacent countries. - Bot. Acta 107: 387-392.

Parvela, A. A. 1930: Oulun läänin viljelyskasvit. Niiden historia ja levinneisyys. I. - Ann. Soc. Vanamo 13(1): 1-334.

Scholler, M. 1994a: Morphologische und chorologische Untersuchungen an Microsphaera palczewskii (Erysiphales). - Feddes Repert. 105: 377-382.

Scholler, M. 1994b: Die Erysiphales, Pucciniales und Ustilaginales der Vorpommerschen Boddenlandschaft. Ökologisch-floristische, florengeschichtliche und morphologisch-taxonomische Untersuchungen. - Regensb. Mykol. Schr. 6: 1-325.

Tikhomirova, I. N. \& Tobias, A.V. 1999: Obligate parasitic micromycetes of the Leningrad region. - Trans. St. Petersburg Nat. Soc. 6(2): 174-196. 